\title{
Sección bibliográfica
}

\author{
Reseñas
}

Agustín Escobar Latapí. Con el su dor de tu frente. Mercado de trabajo y clase obrera en Guadalajara. El Colegio de Jalisco, Guadalajara, 1986, $312 \mathrm{pp}$.

Este libro de Agustín Escobar constituye un buen ejemplo de madurez y consolidación de los estudios sobre mercados de trabajo y clase obrera en el país. Se trata de una obra basada en un exhaustivo trabajo de campo realizado a principios de los años ochenta, y que logra integrar diversas perspectivas teóricas y metodológicas.

Uno de los ejes centrales del libro lo constituye la relación entre segmentación del mercado de trabajo en Guadalajara y segmentación al interior de la clase obrera. Agustín Escobar argumenta y demuestra a lo largo del libro que la presencia de distintos tipos de empresas en la ciudad - denominadas procesadoras, ensambladoras, y un tercer tipo que comparte características de unas y otras-, no lleva a divisiones internas de la clase obrera en diferentes "estratos" de trabajadores. En palabras del autor, la situación anterior

.. . acarrea consecuencias importantes para el análisis del éxito aparente del orden social tapatío en términos de su estabilidad. En mi opinión ésta depende en buena medida de la movilidad intrageneracional y de la falta de criterios claros para la estratificación de la clase obrera local en "subclases" bien definidas. Los individuos corren con distinta suerte, según sus diferentes calificaciones, sus relaciones sociales y los trabajos que consiguen, pero no existen criterios étnicos, raciales o educativos que los dividan en cuanto clase (p. 209).

Otra perspectiva analítica que está presente a lo largo del libro es el interés por demostrar las debilidades y ambigüedades de las categorías formal e informal para entender el funcionamiento de los mercados de trabajo. El autor argumenta que estos conceptos resultan inapropiados para categorizar las empresas, porque aquellas que generalmente se denominan formales no siempre contratan la mano de obra más capacitada ni recurren necesariamente a ascensos internos de la mano de obra ni a controles del ritmo de trabajo mediante prácticas gerenciales. Por su parte, los pequeños talleres artesanales tapatíos, que comúnmente se caracterizan como informales, ofrecen a veces mejores salarios a los varones calificados e incluso llegan a dar la prestación del seguro social. Desde el punto 
de vista del individuo, el tránsito de los empleos formales a los informales es amplio y está bien documentado en la investigación.

El libro ofrece también numerosas aportaciones en torno al conocido efecto que tienen algunas características individuales en el ingreso y la estabilidad en el mercado laboral. De esta suerte, el autor establece que el género constituye en el caso de Guadalajara la característica fundamental. Las mujeres son relegadas a los peores empleos; su trayectoria laboral se encuentra tan nítidamente separada de la de los hombres, que el autor propone conceptualizar esta situación como una de segregación en vez de segmentación como usualmente se le denomina en la literatura sobre el tema. "En lo único que son comparables las trayectorias de hombres y mujeres es en la capacidad de los grupos para cambiar de trabajos formales e informales" (p. 290).

Junto con el género, el origen rural de los trabajadores es el aspecto que deja una huella más patente sobre las trayectorias laborales. Los migrantes rurales permanecen más tiempo en los trabajos menos privilegiados, a excepción de lo que sucede en la manufactura. Asimismo, la puerta de entrada de estos trabajadores a la economía urbana es la industria de la construcción, algo que también se ha hecho evidente en investigaciones sobre la ciudad de México y Monterrey.

La edad juega un papel distinto que las características anteriores sobre la trayectoria laboral de los individuos analizados, pues se comprueba un mejoramiento de sus condiciones de trabajo a medida que ésta avanza. Los jóvenes, por su parte, están más dispuestos a sacrificarse como aprendices con miras a instalarse mejor en el futuro. Por último, la calificación - un aspecto muy trabajado a lo largo de toda la investigación- no ofrecí́ resultados sistemáticos como los mencionados arriba, en parte por las diferencias tecnológicas y de procesos de trabajo involucradas en los diferentes tipos de empresas.

El libro de Agustín Escobar no sólo es rico en resultados científicos como los mencionados hasta aquí, sino que también refleja un compromiso con la realidad analizada y una gran sensibilidad, que se hace evidente en las ricas descripciones sobre los diferentes tipos de empresas. Por ejemplo, al referirse a los pequeños talleres, el autor plantea:

Así pues, aunque formalmente ocultos a la vista, estos talleres son conocidos en todo el vecindario, y un ojo avizor los detecta fácilmente. Existe, claro está, mucha complicidad en el barrio: a toda persona con aspecto foráneo se le hace creer que no hay ningún taller. Esto no significa que se engañe fácilmente a los inspectores de Salubridad, del Seguro Social o del fisco. Sus cateos y descubrimientos forman parte amarga del folklore local, y son paulatinamente aceptados, aun comprendidos, si los inspectores están dispuestos a negociar. La mayoría lo está, ya sea por interés o por consideración. Los funcionarios de los sindicatos caen bajo la misma categoría que los inspectores y suelen actuar de una forma similar. Cuan- 
do no se muestran flexibles sobreviene un verdadero desastre. Se puede destruir un taller de un plumazo, y la vida de la gente se ve dramáticamente afectada. Circulan historias sobre algunos propietarios que sufrieron infartos o simplemente murieron después de una de esas inspecciones (p. 130).

Si analizamos, por último, el libro desde la perspectiva de sus contribuciones teóricas y metodológicas, encontramos numerosos aspectos sobresalientes. El autor desecha de partida que la realidad de Guadalajara tenga que ser analizada a partir de conceptos que él denomina como "subdesarrollados" (la marginalidad constituiría un ejemplo en este sentido). Parte más bien, como ya hemos mencionado, de la dicotomía formal-informal porque la considera útil, y a lo largo de la investigación va mostrando sus deficiencias y limitaciones. A pesar de este esfuerzo, considero que Agustín Escobar es demasiado respetuoso de la dicotomía mencionada, pues no llega a proponer cortes analíticos alternativos. Ésta no es una tarea fácil, pero ya contamos en México con una importante tradición de estudios detallados sobre mercados de trabajo - por lo menos en las grandes áreas metropolitanas-, que merecen ser articulados en un esfuerzo por construir una interpretación propia sobre nuestro acontecer estructural y coyuntural.

En lo que respecta al campo metodológico propiamente dicho, este libro muestra importantes avances y también hace patentes los retos que debemos enfrentar en el futuro. La aportación más valiosa es el empleo de diferentes niveles y unidades de análisis en la investigación. Por un lado, se estudian propiamente las empresas, donde el Agustín Escobar antropólogo produce algunas de sus mejores páginas, describiendo y analizando la empresa Kodak, los talleres de Rodolfo y Chela, el taller familiar de Juan Núñez, o la exitosa pequeña empresa de Trevi. Otro nivel de análisis es el de los obreros mismos y sus trayectorias laborales, donde el autor, según apreciación propia, hace suyo el hábito sociológico por excelencia de reificar el análisis cuantitativo. No obstante, en este último nivel de análisis no siempre se considera importante hacer referencia al contexto de trabajo de los individuos analizado anteriormente, por lo que el lector encuentra alguna dificultad para seguir el desarrollo del pensamiento del autor. No es que no se realice un esfuerzo por integrar los niveles, pero éste no recibe la atención que merece dentro de la obra, y los resultados que se obtienen en este sentido se reportan en un apartado un tanto aislado hacia el final del libro. De todas maneras, las dificultades que encuentra el estudio desde esta perspectiva son comunes a muchos trabajos que se están realizando actualmente en este campo. Su mérito consiste en haber mostrado distintas maneras de enfrentarlas y en señalar la riqueza de los caminos interdisciplinarios. 
Jorge Padua y Alain Vanneph (compiladores). Poder local, poder regional. El Colegio de México y Centre d'Études Mexicaines et Centramericaines (CEMCA), México, 1986, 287 pp.

\section{La territorialidad del poder}

Desde hace varios años, el Centre de Recherches et Documentation sur l'Amérique Latine (CREDAL) de la Universidad de París III está desarrollando una interesante línea de investigación sobre la problemática de los poderes locales en países de 'América Latina como Brasil, Venezuela y México. Considerado como "tema pivote" de una corriente dentro de la investigación social, que convoca a gente de diversas disciplinas (antropólogos, geógrafos, sociólogos, arquitectos urbanistas, etc.), el enfoque y la propuesta metodológica están dirigidos a lograr, descubrir y establecer las distintas, imbricadas y cambiantes articulaciones entre los procesos y fuerzas sociopolíticas y económicas y sus soportes y manifestaciones territoriales, locales-regionales.

Por muchas razones y contradicciones, "lo local" está adquiriendo una particular relevancia en las sociedades capitalistas actuales, centrales y dependientes. En el campo de la investigación social, este retorno al nivel local, al espacio municipal, llega con nuevos aires y perspectivas de análisis, que sacan a este nivel del "paisaje típico" de la comunidad de los estudios culturalistas de la tradición antropológica, y diversifica la identificación de los agentes y las redes de poder local y sus transformaciones hasta un punto nunca alcanzado por los trabajos de los investigadores marxistas, quienes han prestado mayor atención a las estructuras de poder caciquil en las regiones campesinas, o a los grandes aparatos del Estado central.

Esta recuperación del análisis del universo local, para dar cuenta de la complejidad y heterogeneidad de los actores, conflictos y cambios sociales y espaciales, está asociada a un cuestionamiento de la producción de las llamadas "grandes teorías" y sus propios "techos" explicativos, y a una recapitulación para emprender el viraje hacia los estudios de caso, con la perspectiva de construir teorías de "mediano alcance". Es decir que, mediante la progresiva acumulación de conocimiento y discusión de hipótesis y resultados, se puede ir resolviendo el desafío teórico más fuerte que enfrenta esta línea de investigación: la conceptualización del poder local y de las mediaciones e interconexiones que ligan lo local-regionalnacional-internacional de una formación social, en su proceso histórico y diversas coyunturas, y la reflexión teórica sobre la relación entre el poder y el espacio, desde los estudios orientados por la búsqueda de la geografía del poder.

Este libro publica las ponencias presentadas en el seminario sobre poder local y regional que se realizó, con el patrocinio del Centro de Estu- 
dios Sociológicos (CES) de El Colegio de México y el CREDAL, en julio de 1984 en la sede de El Colegio de México. Un primer bloque de artículos corresponde a los trabajos de investigadores adscriptos al CREDAL, en los que destaca el esfuerzo por abordar la constelación de agentes y la trama espacial de poderes, a través de la reconstrucción de los procesos y mecanismos que inciden en la apropiación, organización y distribución de las bases territoriales sobre las que se ejercen las diferentes formas de poder en los municipios y regiones estudiadas. Como señala Daniel Hiernaux en su artículo "Enclave y geografía del poder en Ciudad Lázaro Cárdenas", todavía son escasas las investigaciones que enfocan el estudio de los grandes complejos industriales por el lado del impacto que produce en las relaciones sociales y los núcleos locales de poder existentes, y en los desplazamientos, recambios, alianzas y nuevas estructuras resultantes de las profundas transformaciones espaciales y sociopolíticas ocasionadas por este tipo de proyectos de inversión.

El autor sigue la pista a los diferentes actores institucionales que han participado en la ejecución del proyecto siderúrgico Lázaro Cárdenas-Las Truchas desde mediados de la década de los sesenta hasta la actual. Estos actores - los de mayor poder se encuentran en el territorio local一, son el conjunto de dependencias del gobierno (Sicartsa, Fidelac, empresas paraestatales, Comisión del Río Balsas, IMSS, SEP, etc.), que llegan a la región con decisiones, recursos y criterios de actuación en función de un proyecto de enclave, no sólo económico, según sostiene el autor, sino también político, por lo cual se comprueba "la pérdida de autonomía local de las instituciones, sometidas a las decisiones del más alto nivel del poder central, y el sometimiento de los intereses del desarrollo local y regional a los imperativos de un modelo de desarrollo impuesto por el centro". Este modelo privilegia los intereses extrarregionales pero, de todos modos, genera una nueva dinámica social en la que juegan un papel en la disputa por los espacios de poder y recursos locales las organizaciones sindicales, civiles, religiosas, los partidos políticos y los diversos intereses económicos derivados de las inversiones de capital en la zona.

El artículo de Elsa Laurelli, "El sistema de poder en la producción del espacio urbano-rural: el caso de la microrregión de Río Escondido" y el de Gilíes Fourt, "Poder sindical en una ciudad industrial: el caso de Monclova, Coahuila", responden a esa misma preocupación por conocer a fondo similares situaciones de enclave (el Proyecto carbo-eléctrico de Río Escondido y la implantación de Altos Hornos de México en Monclova, ambos en el estado de Coahuila), el juego de intereses y agentes extralocales y locales que se desarrolla en el curso de las convergencias, divergencias, alianzas y contradicciones que desatan estas inversiones en territorios fronterizos, aislados y escasamente poblados.

En ambos estudios, llama la atención la vulnerabilidad del gobierno municipal ante las demandas de la colectividad, y los conflictos y presio- 
nes que provienen de las organizaciones sindicales (Suterm y Sindicato Minero sección 293 y sección 147), pero también su relativa autonomía como espacio político local para la conformación de alianzas y representaciones que tienen una lógica propia y se apartan en ciertas coyunturas de las decisiones y esquemas definidos por las instancias estatales y centrales de los partidos políticos y sindicatos.

El papel estratégico del gobierno municipal para el control de la distribución de beneficios políticos y económicos, y la ingerencia de los líderes sindicales, son analizados en los artículos de Roland Trabis, "La organización del poder político a escala local: el caso de Nuevo Laredo, Tamaulipas" y Marie-France Prévôt-Schapira, "Espacio petrolero y poder sindical en la costa del Golfo". En el caso de Nuevo Laredo, el autor rastrea la influencia de la Federación de Trabajadores-CTM en los gobiernos municipales desde 1949, cuando su dirigente local llegó a la presidencia municipal; también estudia la ramificación del poder sindical en los diversos ámbitos de la vida municipal y los arreglos para la coexistencia con el otro poder de incidencia local, el "imperio económico" de la familia Longoria en el estado de Tamaulipas. 'Aquí, el autor habla de la coexistencia de dos poderes o "dualidad" de poderes, en el sentido de un acuerdo y reconocimiento implícito y mutuo de los respectivos dominios, para que uno no interfiera y compita con el otro en sus lugares de control y acumulación principales. Esta dualidad se presenta en otros términos de correlación de fuerzas en los conflictos locales en el municipio de Juchitán; Marie-France Prévôt-Schapira y Hélène Rivière d'Arc estudian la resistencia, reivindicación y lucha de tradición étnico-campesina de la Cocei contra el caciquismo, el despojo de tierras, la modernización agrícola de la región y la sujeción de los ayuntamientos a las imposiciones del partido oficial, en el artículo "Poder y contrapoder en el Istmo de Tehuantepec".

Si en los estudios sobre los enclaves industriales se señala el desplazamiento de los gobiernos municipales y la desmunicipalización de sus funciones, que son ejercidas por las dependencias centrales u organismos "desconcentrados", el artículo de Patrice Melé aporta información muy reveladora sobre las dificultades y límites de la planificación municipal del desarrollo urbano, en el artículo "Poder local y urbanización en la ciudad de Puebla". El autor hace una reflexión sobre las instancias, mecanismos e intereses sectoriales que intervienen en la producción del espacio urbano y la dotación de servicios públicos, que transgreden la normatividad de la planificación municipal, se yuxtaponen y traslapan, pero también coinciden en ciertas exigencias o motivaciones de tipo político-económico. Es de lamentar que el CEMCA y el Departamento de Publicaciones de El Colegio de México, encargados del cuidado de la edición del libro, no hayan revisado la redacción en español de este artículo para la mejor comprensión de las ideas del autor. 
Por la brevedad de todos los artículos, muchos elementos de información, análisis y planteamientos teórico-metodológicos quedan desconocidos para el lector, por lo que sería muy pertinente la traducción y publicación en México de las investigaciones completas.

\section{Regiones agrícolas y la urbanización del poder}

Otro grupo de artículos es producto de investigaciones en regiones de alta potencialidad agrícola, en las que los circuitos de poderes locales y regionales, sus transformaciones y emergencia de nuevos actores politicos, se asocian a los cambios en la organización de la producción, financiamiento y comercialización, y al intenso crecimiento urbano de sus principales ciudades. Éste es el caso de Ciudad Mante, en la región del Mante, Tamaulipas, seleccionada por Marielle Pepin Lehalleur (CES) para aplicar una opción metodológica que hace explícita en su artículo con el objeto de analizar "las formas concretas de intercambio político a través de las cuales los grupos sociales campesinos se conforman o se desintegran, en su confrontación con otros sujetos sociales alrededor de la preservación o ampliación de su espacio de reproducción social", y la constitución de los agricultores de la región como sujetos sociales, identificando las distintas áreas de conflicto en los subsistemas agrarios estudiados (el azucarero, el ejidal colectivizado, el parcelario y el empresarial). También es el caso del Bajío zamorano en el estado de Michoacán, acerca del cual el artículo de Gustavo Verduzco (CES), "Poder regional, estratificación social y proceso de urbanización en Zamora, Michoacán”, destaca el cambio en la composición de los miembros del Partido Acción Nacional (PAN), quienes de jóvenes católicos de familias de rancia aristocracia se fueron convirtiendo en portavoces de la pequeña burguesía urbana, que en las elecciones municipales de 1984 ganó el ayuntamiento de la ciudad de Zamora, representando el descontento de estos nuevos sectores urbanos por la ineficiencia y desatención de la gestión estatal.

La huella del pasado y la descentralización del futuro están juntos, pero no mezclados, en artículos, unos (De la Peña; Pastor; Falcón) que, mirando y reflexionando hacia atrás, tratan de encontrar los eslabones sociohistóricos y culturales que le dan razón a la existencia y resistencia del caciquismo, y a la ancestral fragmentación y lucha social por la institución municipal en México. Otro, el de Alain Vanneph y Jean Revel-Mouroz, sobre "La descentralización en Francia", trae una polémica cuestión del presente, que carga el peso de un Estado tan fuerte y agobiadoramente centralizado, que ya le pesa demasiado al propio Estado en Francia y en otros países, como México. La política de descentralización, como reforma del Estado centralizado, implica una serie de medidas, adecuaciones y disposiciones jurídicas, normativas, financieras, administrativas y de 
gestión pública del territorio que, para el proceso francés, los autores logran exponer de una manera concisa pero amplia, no obstante la complejidad y múltiples vaivenes que sabemos caracterizan la realización o intención de echar a andar una política de descentralización. Los autores identifican las principales contradicciones que vienen del papel y situación tan especial e importante que adquiere el gobierno local (municipal) dentro de la política de descentralización. Y hacen una pregunta crucial, que también se plantean muchos otros analistas interesados en esta cuestión: "¿Es la descentralización, en tiempos de crisis, una mera desconcentración de la impopularidad ligada a la gestión de la crisis ya que las autoridades locales no pueden continuar devolviendo la responsabilidad de ciertas situaciones locales hacia el Estado central?"

Es una buena pregunta para estimular a los investigadores a que incorporen dentro de sus proyectos de estudio de la problemática de los poderes locales en México, las tendencias, resultados, manejo y concepciones que se observan en la ejecución de la reforma municipal de febrero de 1983, contenida en las modificaciones y adiciones al artículo 115 constitucional.

Alejandra Massolo

Juan Manuel Ramírez Salz. El movimiento urbano popular en México. Siglo XXI/IIS-UNAM, México, 1986, 224 pp.

Uno de los temas característicos de la investigación urbana de los años ochenta en México, es el correspondiente al estudio de los llamados movimientos sociales urbanos. Como es sabido, las formulaciones teóricas clásicas, de corte marxista, fueron elaboradas principalmente por Manuel Castells y Jordi Borja, bajo cuyas directrices metodológicas se han realizado -no siempre con resultados afortunados-, múltiples trabajos sobre las ciudades latinoamericanas, desde la década pasada.

Si bien los diversos análisis sobre la experiencia del movimiento de pobladores en Chile durante el gobierno de Salvador Allende, por ejemplo, permitieron conocer mejor el carácter emergente de su lucha, sucesivas investigaciones se han planteado nuevas interrogantes y métodos de trabajo. De tal manera, que una de las cuestiones que aún llama la atención en este debate, es acerca de si se trata de una nueva forma de la lucha de clases, a partir de las contradicciones que plantea el desarrollo urbano de tipo capitalista.

El libro de Juan Manuel Ramírez prosigue un esfuerzo de investigación, que desde una perspectiva marxista, han realizado varios estudiosos del tema en nuestro país. Al respecto, hoy resulta todavía paradójico que a más de tres años de haber sido escrito, el análisis sistemático de las 
luchas urbanas y la recuperación de su memoria histórica que se realiza en el libro, apenas esté en sus inicios, como bien se señala en la introducción del mismo. Sin embargo, cabe reconocer la actual diversificación de líneas específicas de investigación y la existencia de nuevos estudios regionales y de caso, entre los cuales pueden destacarse posteriores trabajos del autor.

Para fines de esta reseña, conviene especificar que por movimiento urbano popular se entiende "aquel que está integrado por el proletariado urbano en sentido amplio y que intenta mantener una autonomía del Estado y de la burguesía y definir un programa urbano alternativo al que rige el desarrollo de la ciudad capitalista" (p. 34). Esta concepción, que se distingue del término "movimiento social urbano" -en tanto éste se refiere a las reivindicaciones urbanas interclasistas-, no aclara suficientemente quiénes forman ese proletariado en sentido amplio. En todo caso, cabría verificar mediante un amplio análisis comparativo de distintos movimientos, las características que asume la posición de clase de este sector de las clases dominadas, frente a la burguesía y el Estado capitalista. Dicho sea de paso, está presente a lo largo de este trabajo la tesis que concibe la lucha del movimiento urbano popular (MUP) como elemento básico para comprender el fenómeno de la lucha de clases en México, y en específico la que tiene lugar en las diversas ciudades del país. Así, se llega a afirmar que "todo espacio urbano disputado por las clases sociales, se convierte en un espacio político".

Hechas estas consideraciones generales, pasemos ahora al contenido del texto.

El capítulo I consta de seis breves incisos en los que se hace una caracterización del fenómeno en los siguientes aspectos: los factores clave de la emergencia y desarrollo del MUP; los protagonistas de la lucha, su relación con el Estado y niveles de politización; el tipo de demandas planteadas; su composición socioeconómica o "carácter de clase" de sus integrantes; los adversarios del movimiento y los efectos urbanos y políticos que producen.

Es preciso, sin embargo, reconocer las limitaciones teóricas existentes en la literatura especializada sobre el tema. Por ello, más que una contribución teórica en este sentido, el primer capítulo representa el marco mínimo de análisis y verdadero punto de partída del trabajo. De manera que el mismo término MUP no se asume como categoría acabada, "sino en tanto refleja el nivel de las investigaciones que hasta el presente se han logrado en este espacio de la sociología urbana" (p. 27).

En forma más desarrollada y ampliamente documentado, el siguiente capítulo hace una exposición sistemática del desarrollo de los MUP en México. Si bien se descarta la idea de una aparente "novedad" de las luchas reivindicativas urbanas -el movimiento inquilinario de los años veinte así lo demuestra-, puede decirse que es sólo hasta 1968, con la cri- 
sis del modelo de dominación tradicional, cuando surge en definitiva un movimiento urbano popular organizado e independiente.

La experiencia aportada desde entonces por los MUP ha sido ya recogida en textos anteriores y presentada en esquemas de periodización histórica que dan cuenta de las diversas estapas de organización y lucha de los MUP. Juan Manuel Ramírez hace una cuidadosa revisión de los principales textos que tratan el tema y sobre esta base realiza su propia periodización, entre 1968 y 1983.

Así, se distinguen dos grandes fases y varias etapas en cada una de ellas: la primera, que va de 1968 a 1978, está marcada en sus inicios por el auge de tomas de tierra en diversas ciudades del país al margen del PRI -con lo cual se denota la emergencia y el ascenso de las luchas urbano populares-, y tiene su fin con el repliegue del MUP ante la política represiva del Estado durante los primeros años del gobierno de José López Portillo. La segunda fase (1979-1983) engloba el proceso de recomposición y conformación de la Coordinadora Nacional del Movimiento Urbano Popular (Conamup), hasta las primeras tentativas de articulación intersectorial en 1983, antes del primer paro cívico nacional.

Resulta sumamente útil la reconstrucción histórica del MUP que se presenta a grandes rasgos, así como la política del Estado adoptada ante los conflictos urbanos en las distintas etapas, aun cuando ello deba ser objeto de sucesivas investigaciones que permitan mostrar la variedad de "estilos" de la política urbana estatal.

El origen de la Conamup merece una reflexión aparte si se considera el contexto político nacional, en el que la sociedad civil se halla escasamente desarrollada. Es decir, una explicación unilateral sobre tal fenómeno, que pone el acento en las características materiales del proceso de urbanización capitalista en México, se vuelve insuficiente. Se trata, pues, del surgimiento de una organización de masas, que a juicio del autor, constituye un fenómeno básicamente político. En otras palabras, es necesario repensar la instancia de lo político en el desarrollo capitalista dependiente y, en concreto, la forma en que los movimientos urbanos de las clases dominadas se integran en el contexto de la política a nivel local.*

El capítulo II termina con la exposición de un extenso cuadro que muestra la distribución territorial de las principales organizaciones del MUP, por estado y ciudad, desde Aguascalientes hasta Zacatecas (en orden alfabético). No está suficientemente explicado, por cierto, el desarrollo desigual de los MUP, pues aun cuando para 1983 se localizaban ya en 25 estados, el mayor número de ellos se concentraba en únicamente ocho (Baja California, Distrito Federal, México, Guerrero, Sinaloa, Sonora, Nuevo León y Durango). Por ejemplo, no es constante la relación entre ciuda-

\footnotetext{
* Véanse las conclusiones a las que llega Carlos Nelson Ferreira Dos Santos en su libro Movimientos urbanos no Río de Janeiro, Zahar Editores, Rio de Janeiro, 1981.
} 
des de tipo ỉndustrial y existencia de movimientos urbanos independientes. Aquí sólo cabe en efecto una explicación más precisa a partir de las modalidades que históricamente adopta el sistema político y las relaciones de dominación, a nivel de cada una de las entidades federativas del país. Ello permitiría entender por qué en la actualidad está prácticamente ausente el MUP en Campeche, Aguascalientes o Colima, por citar tres tipos distintos de ciudades medias.

Una visión más completa del fenómeno se obtiene en el capítulo III, que puede decirse representa la parte más sustanciosa de la investigación. Cuatro son las dimensiones que se presentan sobre estos movimientos, en los cuales se pone de relieve su estructura, organización y dinámica politicas. Ciertamente los estudios de caso seleccionados no pretenden ofrecer una muestra sociológica del conjunto de los MUP en México, sino sólo algunas de sus principales expresiones. Cabe decir que esta parte de la obra está fundamentada en un amplio trabajo de campo y de gabinete, que incluye visitas a colonias de Durango, Monterrey y Acapulco a fines de 1982, entrevistas a dirigentes, cuadros y bases de los movimientos, así como análisis de fuentes secundarias y primarias que documentan profusamente la investigación.

El primer estudio de caso corresponde al Consejo General de Colonias Populares de Acapulco (CGCPA), durante los años de 1980 a 1982. Este movimiento resulta sumamente representativo de aquellas organizaciones que, a pesar de tener antecedentes en décadas pasadas, sólo logran conformarse como resultado de una determinada política del Estado que modifica súbitamente sus condiciones de vida y, en particular, su hábitat urbano. Recordemos tan sólo la medida tomada durante la administración de Rubén Figueroa en el estado de Guerrero, de desalojar a 125 mil colonos del puerto de Acapulco, bajo el supuesto de que "contaminaban" la bahía. La respuesta organizada de los colonos fue casi inmediata y una vez integrado el CGCPA logró erigirse en un grupo real de presión y de representación ciudadana independiente frente al aparato de Estado local: el Fideicomiso Acapulco. Finalmente, el CGCPA no logró evitar el poblamiento masivo de Ciudad Renacimiento, pero mostró su capacidad de movilización de amplios sectores de la población, como nunca antes en la historia de las luchas populares locales.

Casi en entero contraste figura el caso del Comité de Defensa Popular (CDP) General Francisco Villa. El CDP es en efecto uno de los movimientos urbanos más consolidados en México. Tuvo su origen unos 20 años atrás, en el Frente Cívico Durangueño de composición pluriclasista y en el Frente Popular de Lucha de 1970 . Aunque no fue hasta 1979 cuando quedó plenamente formado bajo una "estructura de frente amplio" (colonos, ejidatarios, comuneros, comerciantes y transportistas). Desde años atrás, el CDP es considerado como una fuerza política local, de gran influencia en las colonias populares de la ciudad de Durango, frente a la figura de la CNOP del PRI. 
Algo que debe resultar de gran interés para el analista político y motivar nuevas indagaciones cientificas, es la actitud de los MUP frente a los procesos electorales locales, la cual dista de ser inmutable. El CDP de Durango es un botón de muestra al respecto. De un radical rechazo a las elecciones a principios de los años ochenta, pasó a una participación menos que discreta con el PRT en los comicios de 1986 para renovar gobernador, presidentes municipales y diputados locales. Este cambio de actitud se debe en gran parte a los resultados adversos para el CDP, que representó la gestión antipopular del PAN en el municipio de Durango en esos años.

La Unión de Colonias Populares del Valle de México (UCP) es otra de las organizaciones estudiadas y presenta un perfil distinto al CDP, pues se ha constituido orgánicamente en el "sector urbano popular" de una conocida corriente política de izquierda: el Movimiento Revolucionario del Pueblo (MRP) (en actual disolución al fusionarse recientemente al Partido Mexicano Socialista, junto con otras organizaciones y partidos políticos).

La UCP aglutina a varias colonias de municipios conurbados a la ciudad de México y de algunas delegaciones del Distrito Federal, aunque tiene su base principal en la zona metropolitana del Estado de México, actual espacio de confrontación política entre organizaciones oficiales e independientes. Casi desde su formación, en 1979, la UCP ha desarrollado una importante actividad electoral dentro de las colonias populares, que le ha valido serias críticas dentro de la Conamup, a la cual pertenece. El mismo Juan Manuel Ramírez cuestiona en este punto a la organización, por dedicarse fundamentalmente a las actividades electorales -en alianza con el PRT - y descuidar su trabajo con la Conamup. Hay que añadir que en estas circunstancias la UCP ha podido avanzar poco ante los nuevos asentamientos irregulares en municipios como Ecatepec, Chalco o Chimalhuacán, capitalizados en su mayoría por el PRI.

La situación arriba descrita tuvo su climax en el IV Encuentro de la Conamup (1983), al definirse el carácter de su participación, lo cual al no resolverse en dicha ocasión, llevó a la realización por primera vez, de un Encuentro Extraordinario de la Coordinadora en agosto de 1983. Allí la UCP manifestó continuar dentro de la Conamup.

Con respecto a ésta, el autor la concibe como "una coordinadora amplia de masas de tipo frentista que intenta evitar la dispersión del MUP y lograr su avance en un proyecto común" (p. 174). Más adelante, añade que no representa una organización política (partidaria o no), pues no reconoce orgánicamente a ninguna de éstas en su interior. Aquí se hace una amplia exposición a la ideología, formas de lucha y tipos de demandas planteadas por la Conamup, que sintetiza su proyecto político, aun cuando todavía no se deduce de todo ello un programa alternativo del sector urbano popular a la ciudad del gran capital.

El texto finaliza señalando las perspectivas del MUP en los años ochenta. La alternativa más probable, indica el autor, es que la Conamup 
no sea absorbida por los partidos polfticos o por los sindicatos de trabajadores, ni por la propia CNOP, sino que llegue a consolidarse internamente como una instancia sectorial de lucha de las clases subalternas en México.

En el post scriptum de marzo de 1984, aparece una observación importante en torno a la política del Estado en el régimen de Miguel de la Madrid: "la lucha se vuelve, al mismo tiempo, mucho más 'técnica' y más política para el MUP en momentos en que, por otra parte, las condiciones materiales de vida [ . . ] hacen casi imposible para las mayorias la sobrevivencia en las ciudades" (p. 217). Cabe decir que con el terremoto de septiembre de 1985, la política urbana estatal mostró todas sus limitaciones, aun cuando logró finalmente llevar adelante el Programa de Renovación Habitacional Popular. Sin embargo, en la ciudad de México el MUP es hoy distinto que el de hace dos años.

Por último, algunos comentarios criticos generales: si el trabajo representa una verdadera aproximación sociológica a este fenómeno y su dimensión histórica y política en nuestro pafs, el autor pudo haber enriquecido enormemente sus conclusiones y reflexiones finales mediante un mayor análisis comparativo de los estudios de caso. Al respecto sería conveniente ampliar, por ejemplo, el apartado dedicado a "las constantes en las cuatro expresiones del MUP", con algunos esquemas comparativos entre sus rasgos singulares y comunes de las organizaciones.

Por otro lado, aun cuando hay un tema que se señala en diversas ocasiones y no forma parte del cuerpo central del trabajo, merece una investigación aparte: nos referimos a la vieja manera de hacer polf́tica en las colonias populares, por parte de los organismos e instituciones oficiales (CNOP, DIF, Consejos de Colaboración, etc.). Hay que reconocer que los problemas de la reproducción de la fuerza de trabajo en las ciudades son resueltos, en efecto, por varias intermediaciones. He aquí otro factor que frena el avance del MUP y que debe evaluarse en su justa dimensión.

En suma, el texto reseñado es ya de consulta obligada para todos aquellos interesados en la historia y el presente de los movimientos urbanos en México. 
Notas*

VarIos. Se fue a volver. Seminario sobre migraciones temporales en A mérica Latina. PISPAL/ CIUDAD/ CENEP/ El Colegio de México, México, 1986, 595 pp.

En este volumen se recopilan las ponencias presentadas en el seminario sobre migraciones temporales en América Latina patrocinado por PISPAL, CENEP y CIUDAD, realizado en Quito, en noviembre de 1984.

Las ponencias son producto de una diversa gama de perspectivas profesionales, metodológicas y geográficas, sobre el tema de las migraciones temporales. Esa diversidad tiene la ventaja de ofrecer un panorama de la complejidad del tema y de las diversas formas en las que éste puede ser analizado.

Por ejemplo, los análisis en algunos artículos se enfocan desde el punto de vista de la problemática general de la cuestión campesina en América Latina; otros se refieren a la gradual ingerencia del capitalismo en el medio rural, examinan la creciente interrelación campo-ciudad, la expansión de la agricultura sobre áreas nuevas y la demanda estacional de mano de obra de algunos cultivos particulares. También se hace énfasis en la migración como resultado de los cambios en el mercado laboral.

Los trabajos están agrupados de acuerdo con la escala de análisis. En los cinco primeros el énfasis está en los aspectos teórico-metodológicos; los cuatro siguientes se centran en las situaciones nacionales respecto a las migraciones temporales; otros cuatro ofrecen una visión regional de la problemática y los últimos seis se concentran en estudios de caso.

Una característica importante de las ponencias es que la información de los censos está casi ausente como fuente de información y se la utiliza como marco general; diversos tipos de encuestas son las fuentes fundamentales de datos.

Asimismo, los artículos se caracterizan por considerar como unidad de análisis, no al individuo, sino al grupo familiar, para la mejor explicación del sentido y el marco de racionalidad de los movimientos; sin embargo, también se presentan como opciones válidas al grupo local o a la región.

Una de las conclusiones más importantes que puede extraerse de los trabajos presentados, es que la migración temporal en América Latina no se circunscribe a la estacionalidad de los ritmos de producción agrícola. Aunque ese tipo de migración sigue teniendo mucha importancia, se dan

* Sección a cargo de María Elena Muñoz. 
también muchas migraciones ligadas a otras actividades, que no sólo se desarrollan en el medio rural.

Gullermo de la Peña, y Agustín Escobar (comps.) Cambio regional, mercado de trabajo y vida obrera en Jalisco. El Colegio de Jalisco, Guadalajara, 1986, 364 pp., (colección "Estudios Sociales" núm. 1).

Esta publicación reúne los resultados de siete investigaciones enfocadas sobre el mercado de trabajo y el proceso de cambio regional de Guadalajara, con objeto de aclarar algunos aspectos importantes del patrón de crecimiento actual de esa ciudad.

En el primer artículo, de Bryan Roberts, se plantea el marco conceptual general que sirve de introducción al resto de los trabajos.

Guillermo de la Peña analiza en seguida los factores que determinan la articulación regional en torno a Guadalajara y su validez actual, así como las implicaciones del contexto socio-espacial para el mercado de trabajo urbano.

Carlos Alba incursiona después en la evolución y perspectivas del proceso de industrialización de Jalisco y en la importancia de su industria a nivel local y nacional.

Agustín Escobar, por su parte, sostiene que la estructura y el sentido de los cambios que tienen lugar en el mercado de trabajo se derivan de la organización social del trabajo y de los cambios que se generan en ella, para arribar a la distinción de tres tipos de procesos de trabajo. Presenta algunos resultados sobre la segmentación del mercado de trabajo con base en una encuesta realizada entre trabajadores manuales de la ciudad de Guadalajara. La encuesta recopiló información sobre más de 5000 trabajos desempeñados por los informantes, y comprendió a 1300 individuos que laboraban en 55 empresas, pertenecientes a las industrias de la transformación, de la construcción y de los niveles manuales del sector público.

El ensayo de Mercedes González de la Rocha se dedica a analizar la forma en que el grupo doméstico se relaciona con el mercado de trabajo. Sus objetivos son aportar conocimientos sobre la segmentación del mercado de trabajo por medio del estudio de la organización de la unidad doméstica de los trabajadores; dar una definición más apropiada de la unidad doméstica; estudiar el papel de la mujer como trabajadora asalariada y doméstica, y como importante "tejedora" de redes sociales dentro de las estrategias de generación de ingresos, sin aislarla de su contexto social inmediato: la unidad doméstica. La metodología combina técnicas de investigación antropológicas y sociológicas. Se realizaron quince estudios de caso para cubrir diferencias ocupacionales, de tamaño y de etapa del ciclo 
doméstico. Se aplicó un cuestionario a cien unidades domésticas de diferentes zonas y colonias de bajos ingresos. Esta muestra se comparó con la del mercado de trabajo (recogida en el artículo de Escobar mencionado anteriormente) y se encontró que no es atípica y permite dilucidar las características de la población de bajos ingresos.

El siguiente artículo, elaborado por Luisa Gabayet O., se basa en un estudio realizado en el sur de Jalisco y muestra resultados distintos a los dos anteriores. En esa región se da la polarización de los mercados de trabajo debido a la prosperidad de grandes industrias y a la precariedad de las medianas y pequeñas. Se hace un análisis comparativo entre dos grandes empresas situadas en el sur de Jalisco y las textileras localizadas en la zona metropolitana de Guadalajara. La comparación se hace sobre tres aspectos: la historia de las empresas, la acción de los sindicatos y el proceso de trabajo. También se compara, posteriormente, a los obreros y sus unidades domésticas. Para este último tipo de comparación se recurrió a la técnica de entrevistas, escogiéndose a veinte familias de obreros de cada una de las industrias analizadas.

En el último ensayo que se presenta, Lorenza Villa Leven analiza la calificación del obrero y del técnico, y su vinculación con el mercado de trabajo en la industria de transformación en Jalisco. El objetivo es dilucidar si las empresas, al seleccionar la mano de obra, toman en cuenta sólo factores técnicos o si también influyen factores sociales. Se analiza después la influencia de las necesidades técnicas de la producción sobre la planeación educativa y los factores que participan en la definición de las especialidades técnicas impartidas en las escuelas de nivel medio básico y medio superior. El análisis está basado en la encuesta industrial aplicada en 1981 a 1244 establecimientos industriales, además de entrevistas a dirigentes patronales, empresarios, jefes de personal y maestros.

Pedro Pírez. La población y el estudio de lo urbano-regional en América Latina. Revisión de los aportes del PISPAL. PISPAL El Colegio de México, México, 1986, 125 pp.

La secretaría ejecutiva del Programa de Investigaciones Sociales sobre Población en América Latina (PISPAL) encomendó al autor la elaboración de este libro, que tiene como objeto evaluar la aportación del PISPAL a la temática urbano-regional y a la problemática poblacional.

La evaluación se realizó mediante la revisión de 19 investigaciones auspiciadas por PISPAL sobre distintos temas interrelacionados: la distribución territorial de la población, las migraciones, la urbanización, el desarrollo regional y los mercados de trabajo.

El libro está conformado de la siguiente manera: presentación, prefacio e introducción general y tres apartados. La primera parte presenta los 
resultados de los trabajos analizados con una descripción de sus características fundamentales. De ellas el autor extrajo un conjunto de elementos conceptuales y empíricos ordenados en relación con el eje temático que se propone, para posteriormente permitir su integración. El criterio de ordenación se orienta hacia tres núcleos fundamentales: primero se presentan los aspectos directamente vinculados con el análisis de la población y sus dimensiones o vinculaciones territoriales; en segundo lugar se muestran los enfoques que analizan a la población desde la perspectiva de los mercados de trabajo y por último se presentan los intentos globales para el estudio de los procesos de la configuración territorial en América Latina.

En la segunda parte se propone un ordenamiento que dé lugar a la problemática de la configuración territorial en América Latina, entendida como un conjunto de procesos que, integrando el proceso global del desarrollo de cada sociedad, va determinando la distribución de las actividades y la población en el territorio.

La tercera parte propone un esquema general que sistematiza la problemática, determinando niveles de análisis y temáticas de estudio. Posteriormente se presentan los materiales de las investigaciones auspiciados por PISPAL teniendo en cuenta el nivel global de análisis, los niveles de mediación y los niveles concretos regionales. De esta forma, se propone un sistema de conocimiento que puede permitir organizar la investigación.

Jorge Martínez Manatou (editor). Planificación familiar, población y salud en el México rural. Instituto Mexicano del Seguro Social, México, 1986, 477 pp.

El objetivo de esta publicación del IMSS es contribuir al conocimiento de algunos aspectos demográficos y de salud de la población rural mexicana relacionados con la planificación familiar.

La mayor parte de la investigación está basada en la información obtenida en la Encuesta Rural de Planificación Familiar realizada en 1981, y en otras encuestas nacionales anteriores.

El libro consta de una introducción y 10 capítulos. En el primero se hace una descripción general del ámbito rural, en donde se destaca que las causas de los principales riesgos y daños a la salud de la población son un ambiente insalubre y una alimentación deficiente, así como una conducta reproductiva que determina un número excesivo de embarazos iniciados a edades tempranas y demasiado seguidos.

Los servicios de planificación familiar en el medio rural se abordan en el segundo capítulo; allí se resume la historia de ese tipo de servicios, iniciados a mediados de la década de los sesenta, y de su paulatina am- 
pliación; también se describen las principales estrategias que ha seguido el IMSS para ampliar y consolidar los servicios de planificación familiar.

Para evaluar las repercusiones y los efectos de la planificación familiar en la estructura y dinámica de la población, se analiza en el tercer capítulo la evolución de la fecundidad y de la mortalidad. En particular, se destaca la elaboración de la tabla abreviada de vida por sexo para la población rural, que se construyó a partir de las estadísticas vitales y los datos de las encuestas.

El cuarto capítulo se dedica a profundizar en el análisis de la fecundidad y sus tendencias en el medio rural, mediante el estudio de cuatro variables intermedias: matrimonio, anticoncepción, aborto y lactancia.

La importancia de la relación existente entre los niveles de fecundidad y la organización económica de las familias en diferentes grupos es el objeto de estudio del capítulo quinto.

Más adelante se analiza la influencia de la disponibilidad y la accesibilidad de los métodos anticonceptivos, el conocimiento y uso de ellos por parte de las mujeres relacionándolo con algunas variables sociodemográficas. Después se estudian los niveles de cobertura de la atención materno-infantil y la influencia de los factores biológicos y sociales en la mortalidad infantil.

El penúltimo capitulo se orienta al estudio del proceso de comunicación con respecto a la planificación familiar; entre la población rural, se consideran las estrategias de comunicación educativa, utilizando un modelo teórico de cambio de conducta.

Por último, se presenta un resumen general de la obra y las principales conclusiones de cada capítulo. Entre éstas destacan: a) los riesgos y los daños a la salud de la población son de mayor magnitud en el medio rural; b) para lograr el mejoramiento de la salud en ese medio, es necesario desarrollar un modelo de atención primaria y de fomento de la salud, acorde con los problemas y necesidades de la población campesina; c) es indispensable retomar las experiencias obtenidas en el transcurso de la evolución de los servicios de salud a fin de asegurar la continuidad de las acciones; d) hasta 1969 las mujeres que vivían en unión libre en localidades con menos de 20 mil habitantes no controlaban su fecundidad; sin embargo, para 1981 se registra un descenso en la tasa global de fecundidad en localidades con menos de 2500 habitantes; e) la esperanza de vida al nacer es menor en el medio rural que la estimada a nivel nacional, lo que significa una diferencia de más de seis años en cuanto a la mortalidad en las zonas rurales con respecto al nivel nacional; fi la reducción de la fecundidad se ve obstaculizada por las condiciones de vida desfavorables de la población rural; g) la disponibilidad de métodos es el factor que ha influido en mayor medida en el incremento de la práctica anticonceptiva observado en años recientes en las áreas rurales. 
Mario Carrillo Huerta (compilador). Los programas regionales de empleo en México. El Colegio de Puebla, Puebla, 1986, 423 pp.

La investigación que se presenta en esta publicación tiene como objetivo examinar y evaluar los programas regionales de empleo a corto plazo que formaron parte de las medidas de ajuste a la crisis financiera y económica de 1982, y su aplicación en el estado de Puebla.

Los objetivos del programa eran proteger el empleo y la planta reproductiva mediante la asignación de gasto público entre grupos sociales y zonas desprotegidas económicamente, de manera que la política macroeconómica anti-inflacionaria incidiera lo menos posible en esos grupos y zonas. El programa incluía más de $\mathbf{5 0}$ tipos diferentes de proyectos que se agruparon, para efectos de la evaluación, en siete grandes grupos: a) servicios a colonias populares y comunidades (dotación de servicios públicos y urbanización); b) proyectos productivos (construcción de unidades de producción); c) proyectos de infraestructura básica (construcción, rehabilitación o ampliación de obras de infraestructura); d) becas de capacitación para trabajadores desempleados; e) servicio social obligatorio de pasantes; f) becas a hijos de trabajadores, y g) programas de integración de la mujer al desarrollo.

Como metodología se eligió realizar un análisis comparativo de los impactos de los diferentes programas, de acuerdo a sus características de equidad social y un análisis de su eficiencia en el logro de los objetivos asignados. Para ello se aplicaron ocho cuestionarios a diferentes grupos, que comprendieron unas 2,700 entrevistas.

La publicación consta de cinco partes y un apéndice metodológico. La primera parte se presenta en dos capítulos; uno sobre el marco general del desarrollo, el desempleo y la política económica, y otro en el que se reseñan artículos dedicados a la evaluación de programas de generación de empleo en países subdesarrollados.

La segunda parte incluye tres capítulos que esbozan la problemática económica actual de México.

En la tercera parte se enfoca el análisis sobre el nivel estatal en dos capítulos; uno presenta el marco macroeconómico y del empleo en el estado de Puebla y el otro presenta la metodología del estudio del impacto de los programas regionales de empleo en Puebla.

La cuarta parte expone los resultados de la investigación en cinco capítulos. En ellos se analizan los efectos inmediatos en el empleo, el ingreso y el bienestar; se discuten los efectos de corto y mediano plazos; se estudia el efecto regional de los programas en el estado; se hace la evaluación administrativa de los programas y, por último, se presenta la evaluación global que incluye aspectos de fondo y de forma.

En la quinta parte se dan a conocer, en un capítulo, las conclusiones 
generales del estudio y recomendaciones metodológicas y de política. Aquí se destaca que, de acuerdo a la metodología adoptada, la efectividad de los programas regionales de empleo fueron positivos y considerables.

El apéndice metodológico incluye cinco apéndices: los cuestionarios aplicados; la metodología para calcular las diferenciales de ingreso real; la metodología seguida en el diseño de la muestra para el estudio de los impactos de los servicios a colonias populares y comunidades; la evaluación de costos y beneficios de dos proyectos de infraestructura básica y la metodología para estudiar el impacto regional de los programas (el cambio y la participación).

Carmen Luz Latorre y Axoo Yonemura. Formation of Urban Low Income Class and Education: Chile and Mexico. JRP Series, núm. 50, Institute of Developing Economies, Tokio, 1986, 225 pp.

Esta publicación recoge el reporte de una investigación, realizada en Chile y en México, cuyo objetivo fue estudiar el proceso de formación de los grupos urbanos de bajos ingresos y el papel que desempeña la educación en sus perspectivas de lograr mayores ingresos y bienestar.

Los autores consideran que la relación positiva entre la educación, la productividad y el bienestar que ha sido el supuesto del que partían muchos investigadores en el pasado, se ha visto cuestionada por la experiencia observada en las últimas décadas.

Tanto para México como para Chile, la investigación se conduce en dos niveles. Primero se aborda el comportamiento de variables como población, ingreso, urbanización, empleo, migración, distribución del ingreso y servicios educativos; de esta manera se definen los sectores urbanos de bajos ingresos en relación a la estructura social y económica en la que se ubican. Posteriormente, en otro nivel, se estudian las características socioeconómicas de los sectores urbanos pobres, en especial su forma de inserción en el mercado de trabajo, identificando los factores que determinan o afectan la oferta y la demanda de empleo. Se analizan especialmente las características de escolaridad de la fuerza de trabajo y los efectos que la educación ejerce sobre las oportunidades de mejoramiento económico y social de esos sectores.

La investigación está basada en diversas fuentes: censos, encuestas de ocupación, estudios sobre migración, pobreza y áreas urbanas marginales, así como en las encuestas que se levantaron en San Antonio de Barnechea (Santiago de Chile) y en ciudad Nezahualcóyotl (México), que los autores consideran barrios representativos de la extrema pobreza.

Las principales conclusiones señalan que la gente pobre es más numerosa en términos absolutos a pesar de las altas tasas de crecimiento económico alcanzadas en las últimas décadas y que está integrada a las 
relaciones económicas, pero se encuentra marginada en lo que a distribución de la riqueza se refiere; de esta forma, las nuevas generaciones tienden a reproducir la situación de empleo de sus padres.

A pesar de que ha habido una fuerte expansión de los servicios educativos, el acceso a la educación sigue siendo muy selectivo. Así, la gente pobre ha logrado incrementar sus niveles de escolaridad, pero esa mayor capacitación no ha influido favorablemente para que logren un mayor nivel de bienestar. Los pobres tienden a ser más pobres con el tiempo.

Elena Zúñiga, Daniel Hernandez, Catherine Menkes y Carlos Santos. Trabajo familiar, conducta reproductiva y estratificación social. Un estudio en las áreas rurales de México. Instituto Mexicano de Seguridad Social, México, 1986, 226 pp.

El libro presenta una investigación sobre los principales aspectos de la conducta reproductiva de la población rural de México, cuyas características específicas se desprenden de las relaciones sociales y de producción mediante las cuales los individuos logran su mantenimiento y remplazo.

El estudio se basa en información proveniente de la Encuesta Rural de Planificación Familiar de 1981; se definen como rurales aquellas localidades que según el Censo de Población de 1970 contaban con menos de 2500 habitantes. La encuesta abarca 8103 hogares y son 44794 las personas entrevistadas.

La publicación consta de una introducción, cinco capítulos y conclusiones generales. En el primer capítulo se presentan las teorías que sirven de marco conceptual y se plantean las hipótesis a seguir; en el segundo se presentan los criterios metodológicos que se siguieron para la conformación de la estructura social y de otros indicadores relacionados con la organización y el ciclo vital de las familias; en el tercero se muestra la organización del trabajo de los distintos grupos sociales considerados y se analizan los factores que determinan la participación económica de los miembros de la familia; en el cuarto se enfoca la relación entre fecundidad y organización del trabajo, considerando, asimismo, otros factores socioeconómicos que influyen en la conducta reproductiva y el comportamiento de las variables intermedias de la fecundidad; el último capítulo investiga la relación entre las distintas condiciones de producción agrícola y la organización del trabajo y la conducta reproductiva.

Las conclusiones más importantes indican que aunque tradicionalmente se ha considerado que la alta fecundidad que prevalece en el medio rural encuentra su explicación en la importancia que tiene el trabajo de los miembros de la familia para obtener un ingreso que les permite subsistir, esta investigación demuestra que existen diferencias que no se asocian con la forma en que se utiliza el trabajo familiar. Así, los mayores niveles 
de fecundidad no corresponden a los dos grupos de mayor participación económica familiar.

Asimismo, se plantea que la integración de los diversos elementos que se desprenden del proceso de desarrollo capitalista favorecen la transformación de la conducta reproductiva hacia familias más reducidas. También se concluye que puede esperarse que el mejoramiento de las condiciones de vida influya en la reproducción con una tendencia hacia la reducción de la fecundidad, siempre y cuando se mejore la oferta de métodos anticonceptivos para esta población.

Diana R. Villarkeal y Víctor Castañena. Urbanización y autoconstrucción de vivienda en Monterrey. Claves Latinoamericanas, México, 1986, 202 pp.

La investigación que se presenta en este libro forma parte del proyecto " $\mathrm{La}$ autoconstrucción de vivienda en México" que inició el Centro de Ecodesarrollo, en 1981, en ocho ciudades, bajo la dirección de Jorge Legorreta.

El objetivo de la investigación es el análisis de los procesos de autoconstrucción y urbanización en el área metropolitana de Monterrey (AMM), considerando que éstos expresan la desigualdad social que ha conducido a la mayoría de la población a soluciones individuales para afrontar el problema habitacional.

El libro consta de una introducción, cuatro capítulos, conclusiones y dos anexos, uno que presenta información sobre las colonias estudiadas y otro que incluye cuadros estadísticos.

En el primer capítulo se hace referencia a la relación entre el proceso de industrialización y urbanización del AMM; se analiza también la estructura ocupacional, la distribución del ingreso y el nivel de vida.

En el segundo se presentan las cinco formas de gestión que han permitido el acceso a la tierra y a la vivienda a los grupos populares. Estas diversas formas de gestión están clasificadas de acuerdo a su carácter político y legal, y a la forma de relación con las instancias oficiales.

El análisis de las características de la población estudiada es el tema que trata el tercer capítulo. En él se destaca que la estabilidad en el empleo y las fuentes de recursos son factores determinantes para la autoconstrucción.

El último capítulo aborda el ingreso familiar y el empleo como factores que influyen en la participación del usuario en la construcción de la vivienda; también se ocupa de la participación de los colonos en las obras colectivas y los servicios urbanos.

Los dos últimos capítulos tienen como fuente de datos la encuesta realizada en seis colonias y tres fraccionamientos, con un total de 187 entrevistas. 
Las principales conclusiones apuntan: que el fuerte proceso de urbanización de Monterrey tuvo su origen en las actividades secundarias; que a partir de la década de los años sesenta la proliferación de asentamientos irregulares se convirtió en la principal causa de la expansión territorial; que en las soluciones al problema habitacional la iniciativa de los sectores populares en particular la del Frente Popular Tierra y Libertad ha sido decisiva; que la gestión pública, si bien permitió el acceso masivo a la tierra y a la vivienda (Fomerrey y el Plan Tierra Propia), se caracterizó por sus prácticas de manipulación política de las necesidades sociales, por lo que la solución al problema habitacional está condicionada a la subordinación política hacia el PRI o al Estado (así, la lógica operativa de los programas de fomento a la autoconstrucción de la vivienda y la regularización de la tenencia de la tierra tuvo como interés fundamental preservar el control de las organizaciones populares de colonos); que la autoconstrucción es una solución tanto para los migrantes como para los nativos del AMM, siendo el factor de mayor peso, según los resultados de la encuesta, la estabilidad en el empleo y, finalmente, que los autoconstructores están incorporados a las actividades industriales, destacándose que dentro de ellas participan grupos sociales con ingresos medios y empleos estables, lo que revela un proceso de deterioro y pauperización, dejando de ser así la autoconstrucción un proceso exclusivo de las clases sociales más deprimidas. 
